\section{Hemifacial spasm: treatment by posterior fossa surgery}

SIR: I write regarding the papers which appeared recently in your journal concerning the treatment of hemifacial spasm by Fabinyi and Adams, 1978;41:829-833, and Kaye and Adams, $1981 ; 44: 1100-$ 1103. I submit that these investigators have not fully understood the concept of vascular compression at the root entry zone of the facial nerve and have been performing the same neurolysis (using the surgical binocular microscope) that Gardner pioneered without magnification many years ago. ${ }^{1}$

It is known from the work of Sunderland $^{2}$ that arterial loops, usually branches of the anterior inferior cerebellar artery, are seen coursing around and through the seventh and eighth nerve bundle in two thirds of cadavers. These vessels are rather distal on the nerves. They are not causal of hemifacial spasm. The blood vessel causative of hemifacial spasm is located at the point where the facial nerve exits the brain stem. One of the authors of one of the above papers with whom I have discussed the operative techniques used in this series gives me to understand that the presence or absence of brain stem root entry zone vascular compression was not evaluated in these patients. It is well known that any manipulation or trauma to the facial nerve will stop hemifacial spasm temporarily and occasionally for a prolonged period of time. Analysis of the 1981 series of 16 patients reveals that of the nine patients followed for two years or more, two had enough trauma to have postoperative weakness and three others suffered recurrence of their symptoms. Of the four arteries which were identified in the 16 patients, my presumption is that only possibly the vertebral artery in case 1 was causal of the spasm. In my experience of over 350 operative patients, I have unfortunately seen that inadequate vascular decompression of the nerve without trauma to the nerve is obvious postoperatively because the spasm persists unchanged. Reoperation was necessary in 13 patients in my series. The data from 229 patients have been summarized. ${ }^{3}$ Blood vessels and occasionally tumours were found in all patients at the root entry zone of the nerve. Over $70 \%$ of the persons had some spasm postoperatively, which gradually disappeared. A more detailed study, with long-term follow-up of these patients, is in preparation.

PETER J JANNETTA
Presbyterian-University Hospital, Room 9402, 230 Lothrop St, Pittsburgh, PA 15213, USA

\section{References}

' Gardner WJ. Concerning the mechanism of trigeminal neuralgia and hemifacial spasm. $J$ Neurosurg 1962;19:947-957.

2 Sunderland S. Neurovascular relationships and anomalies at the base of the brain. $J$ Neurol Neurosurg Psychiatry 1948;11:243-247.

3 Jannetta PJ. Neurovascular compression in cranial nerve and systemic disease. Ann Surgery 1980;192:518-25.

\section{Adams and Kaye reply.}

SIR: We are pleased to have the opportunity to respond to Professor Janetta's claims that neither do we understand his concept of vascular compression at the root entry or exit zone as a cuase of hemifacial spasm and trigeminal neuralgia, nor did we look for such compression at operation. He gives no evidence for his first contention and his latter accusation is based on a conversation in Australia with a former colleague who assisted at only two operations for hemifacial spasm, one four years and the other at least one year before the alleged conversation took place. Hardly an impressive basis for his comments. We suggest he misconstrues and perhaps misuses the politeness with which visitors and their ideas are normally received.

We have, of course, read Janetta's papers carefully and if he had returned the compliment he would see in the first of our papers $^{1}$ a precise description of our technique, which involves retracting the flocculus, exposing the choroid plexus of Luschka's foramen, identifying the vein of the lateral recess, so as to carefully see the junction of the 7th and 8th cranial nerves with the brain stem. We expected to see vascular compression, but have failed to find evidence of this in most of our patients, and so report it. Likewise, we failed to find this in trigeminal neuralgia and a paper describing our findings in fifty-seven patients has been accepted for publication. $^{2}$

As for Jannetta's concept, we understand, but we do not agree. It is perhaps appropriate that we should say why: (1) Jannetta claims arterial and venous compression at the root exit or entry zone causes both trigeminal neuralgia and hemifacial spasm. Despite Jannetta's claims to the contrary, the majority of observers find no sensory loss in trigeminal neuralgia, yet slight facial weakness is almost the rule with long standing hemifacial spasm. It is improbable that the same mechanism causes loss of function in one, but not in the other. (2) Not infrequently do we find such vessels adjacent to root exit or entry zone in patients without hemifacial spasm or trigeminal neuralgia. We see these adjacent to other cranial and spinal nerves in asymptomatic patients. We are aware Jannetta claims vertigo, tinnitus and essential hypertension can be caused by a similar mechanism, but are unaware of similar claims for the motor nerves of the eye, spinal nerves, etc. We note Jannetta ${ }^{3}$ cannot explain why "abnormal" vessels are seen in asymptomatic patients. (3) Jannetta ${ }^{3}$ claims the brain "sags" with age, thus causing veins to compress cranial nerves. He gives no evidence, and we know of no evidence for this. It would require the supratentorial cranial nerves to elongate. Do they? He also claims veins, coagulated and divided, may "recollateralize" and cause a recurrence of symptoms. We find all this too speculative to accept. (4) Jannetta ${ }^{4}$ states that when the patients is in the lateral decubitus position vessels move 1-2 $\mathrm{mm}$, away from the nerve root. Thus he claims patients may abort trigeminal neuralgia by lying in such a position. Why not hemifacial spasm too? We do not find this with our patients with trigeminal neuralgia. We operate on these patients in this position, so why does not Jannetta suggest that this is the reason why we fail to see "his" vessels, rather than making the somewhat wild claim that we do not properly look? Rotating patients during operation has not, however, revealed such movement of vessels to us. (5) Jannetta attempts to prove his concept by comparing asymptomatic cadaver brains (sliced through the mid-brain) with videotapes, movies and photographs (all two dimensional; why not use the surgeons operation note?; how were the patients selected) from patients with trigeminal neuralgia. The authors conclude that there is "a significant" difference of arterial (not venous) compression between the two groups, but curiously state they can not draw "casual inferences" from the data. It seems that Jannetta is so doing. (6) Morley suggests "it only requires one case in which a nerve is not touched by a vessel (or tumour etc.) to discredit the hypothesis entirely". Jannetta's letter fails to state that in the article he refers to, ${ }^{3}$ two of his seventeen patients with glossopharyngeal neuralgia, had no abnormal vessels (or other lesions) at operation. We regret the lack of long 
term follow up in any of the 695 patients reported in the paper Jannetta refers to in his letter. (7) Gradual improvement in the post-operative period does not necessarily support Jannetta's concept. We see such improvement, and assume this is due to progressive fibrosis around the nerve root. Recently one patient developed a mild facial weakness ten days after operation, which completely recovered over the following three months. (8) Although we note vascular compression in a minority of our patients, we are still not sure if this is significant. Certainly, there is no difference in our results for hemifacial spasms in patients with, and without, vascular compression at the root exit or entry zone.

"Root exit or entry zone vascular compression is frequently subtle", writes Jannetta. ${ }^{3}$ "Well trained neurosurgeons may not appreciate up to $30 \%$ of the abnormalities early on in their experience". The inference is also subtle; that to be a well trained and experienced neurosurgeon you have to see these abnormalities. Anderson ${ }^{7}$ warned against this approach, and highlighted the eventual outcome.

We do agree with Jannetta that wrapping or decompression (call it what you will) of the facial nerve is the best operation for hemifacial spasm. We believe the operation works by persistent, but gentle trauma and fibrosis around the nerve. How does Jannetta disprove our concept? But for the patients it matters not how it works, except that it does. Jannetta deserves credit for popularising the posterior fossa approach for hemifacial spasm.

CBT ADAMS Consultant Neurosurgeon ANDREW H KAYE Senior Registrar Neurosurgery The Radcliffe Infirmary, Oxford, $O X 26 H E, U K$

\section{References}

'Fabinyi G, Adams CBT. hemifacial spasm treatment by posterior fossa surgery. JW Neurol Neurosurg Psychiatry. 1978;41:829-33.

2 Adams CBT, Kaye AH, Teddy PJ. The treatment of trigeminal neuralgia by posterior fossa microsurgery. In press $J$ Neurol

Neurosurgery Phychiatry.
${ }^{3}$ Jannetta PJ. Neurovascular compression in cra nial nerves and systemic disease. Ann Surg. 1980;192(4):518-25.

4 Jannetta PJ. Treatment of trigeminal neuralgia by suboccipital and transtentorial cranial operations. Clin Neurosurg 1976;24:538-듬 49.

s Haynes St, Jannetta PJ, Zonio DS. Microvascu-

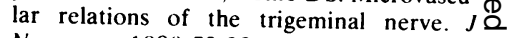

Neurosurg 1980;52:381-6.
- Morley TP. Current Controversies in Neurosurgery. WB Saunders

7 Anderson Hans C. Fairy Tales: The Emperor's New Clothes. 1977;119. Victor Gollancz. 